\title{
Dabigatran Potentiates Gemcitabine-Induced Growth Inhibition of Pancreatic Cancer in Mice
}

\author{
Kun Shi, ${ }^{1}$ Helene Damhofer, ${ }^{2}$ Joost Daalhuisen, ${ }^{1}$ Marieke ten Brink, ${ }^{1}$ Dick J Richel, ${ }^{3}$ and C Arnold Spek ${ }^{1}$ \\ ${ }^{1}$ Center for Experimental and Molecular Medicine, ${ }^{2}$ Laboratory for Experimental Oncology and Radiobiology, Center for \\ Experimental Molecular Medicine and ${ }^{3}$ Department of Medical Oncology, Academic Medical Center, University of Amsterdam, \\ The Netherlands
}

\begin{abstract}
Pancreatic cancer is one of the most lethal solid malignancies, with few treatment options. We have recently shown that expression of protease activated receptor (PAR)-1 in the tumor microenvironment drives the progression and induces the chemoresistance of pancreatic cancer. As thrombin is the prototypical PAR-1 agonist, here we address the effects of the direct thrombin inhibitor dabigatran on pancreatic cancer growth and drug resistance in an orthotropic pancreatic cancer model. We show that dabigatran treatment did not affect primary tumor growth, whereas it significantly increased tumor dissemination throughout the peritoneal cavity. Increased dissemination was accompanied by intratumoral bleeding and increased numbers of aberrant and/or collapsed blood vessels in the primary tumors. In combination with gemcitabine, dabigatran treatment limited primary tumor growth, did not induce bleeding complications and prevented tumor cell dissemination. Dabigatran was, however, not as efficient as genetic ablation of PAR-1 in our previous study, suggesting that thrombin is not the main PAR-1 agonist in the setting of pancreatic cancer. Overall, we show that dabigatran potentiates gemcitabine-induced growth inhibition of pancreatic cancer but does not affect primary tumor growth when used as monotherapy.
\end{abstract}

Online address: http://www.molmed.org

doi: $10.2119 / \mathrm{molmed} .2016 .00214$

\section{INTRODUCTION}

Pancreatic ductal adenocarcinoma is one of the most lethal solid malignancies and is associated with a high propensity for local invasion and distant metastases (1). Despite improvements in the treatment of cancer in general, the overall 5 -year survival rate of pancreatic cancer remains less than $5 \%(2)$ and overall mortality approaches $99 \%$ (3). The high mortality results from the majority of patients presenting with locally advanced and/or metastatic disease, which is rapidly progressive and inevitably fatal. Less than $20 \%$ of patients undergo surgical resection, which is associated with improved 5-year survival rates of around $15-20 \%$. However, the majority of this selected group of patients eventually also succumb to metastatic disease (4).

Protease activated receptor (PAR)-1 (also known as coagulation factor II receptor) is a seven-transmembrane G-coupled receptor that is activated by proteolytic cleavage of the N-terminal extracellular region, thereby releasing a novel tethered ligand that interacts with the body of the receptor to induce transmembrane signaling (5). Expression

Address correspondence to C Arnold Spek, Center for Experimental and Molecular Medicine, H2-215, Academic Medical Center, Meibergdreef 9, 1105AZ, Amsterdam, The Netherlands. Phone: + 3120 5668750; Fax: + 3120 6977192; E-mail: c.a.spek@amc.nl Submitted October 26, 2016; Accepted for Publication January 24, 2017; Published Online (www.molmed.org) February 6, 2017.

Yyivi

Feinstein Institute

for Medical Research Northwell Health

levels of PAR-1 are increased in several cancer types, such as breast and lung cancer (6-8). Moreover, these increased PAR-1 levels are associated with disease progression and reduced overall survival in breast and lung cancer patients $(9,10)$.

Recently, we showed that genetic ablation of PAR-1 from the pancreatic tumor microenvironment limited tumor growth and metastasis in a murine orthotopic pancreatic cancer model (11). Indeed, both tumor volume and weight were reduced around 50\% in PAR-1-deficient mice as compared with wild-type mice, whereas the percentage of mice with metastasis in distant organs was reduced from 100\% in wild-type mice to $12.5 \%$ in PAR-1deficient mice. Even more strikingly, PAR-1 deficiency potentiated gemcitabine-induced tumor regression and completely abolished tumor growth in six out of eight mice.

Thrombin, the central enzyme in the coagulation cascade, is the prototypical 
PAR-1 agonist $(12,13)$, and it is thus tempting to speculate that thrombin is a key driver in pancreatic cancer growth, metastasis and drug resistance. The potential importance of thrombin in pancreatic cancer is underscored by the fact that pancreatic cancer is frequently associated with thrombotic complications, suggesting that thrombin levels are high in pancreatic cancer patients. Moreover, thrombin induces pancreatic tumor growth and invasion in in vitro model systems (14).

Dabigatran etexilate, the pro-drug of dabigatran, is a peptidomimetic reversible and competitive direct thrombin inhibitor (15-18) used in clinics for the prevention of venous thromboembolism after elective hip or knee arthroplasty and stroke in patients with atrial fibrillation (19-22). In the current study, we employed dabigatran etexilate in an orthotopic pancreatic cancer model to challenge the importance of thrombin in pancreatic cancer growth and drug resistance, and we hypothesized that dabigatran would potentiate gemcitabine-induced growth inhibition of pancreatic tumors.

\section{MATERIALS AND METHODS}

\section{Animals}

Eight-week-old wild-type C57Bl/ 6 mice (32 mice in total) were purchased from Charles River. All mice were maintained according to institutional guidelines. Animal procedures were carried out in compliance with the Institutional Standards for Humane Care and Use of Laboratory Animals. The Animal Care and Use Committee of the Academic Medical Center (Amsterdam, the Netherlands) approved all experiments (permit no. DIX102046). Four groups with eight mice per group were used in the experiment.

\section{Cell Culture and Cell Lines}

Murine pancreatic cancer cells (Panc02; kindly provided by Dr. Schmitz, Universitätsklinikum Bonn) were maintained at $5 \% \mathrm{CO}_{2}$ and $37^{\circ} \mathrm{C}$ in Roswell Park Memorial Institute medium 1640 (Invitrogen) supplemented with $10 \%$ fetal bovine serum (FBS); Lonza), 1\% penicillin-streptomycin and 1\% L-glutamine (Lonza). Tumor-associated fibroblasts were isolated from a patient-derived xenograft and grown in Iscove's Modified Dulbecco's Medium (Lonza, BioWhittaker) supplemented with $10 \%$ FBS, $1 \%$ penicillin-streptomycin and 1\% L-glutamine.

\section{Conditioned Medium}

Tumor-associated fibroblasts were serum starved for $2 \mathrm{~h}$, after which they were stimulated with thrombin (1 U/mL) or saline. After $24 \mathrm{~h}$, medium was collected, sterilized through a $0.2 \mu \mathrm{m}$ filter and stored at $-20^{\circ} \mathrm{C}$.

\section{Orthotopic Pancreatic Cancer Model}

Mice were subjected to an orthotopic model of pancreatic cancer essentially as previously described $(23,24)$. Briefly, confluent cultures of Panc02 cells were detached by TrypLE ${ }^{\mathrm{TM}}$ Select (Invitrogen) and pelleted at $340 \mathrm{~g}$ for $5 \mathrm{~min}$, washed twice in phosphate-buffered saline (PBS) and resuspended in PBS. Tumor cells $\left(4 \times 10^{5}\right.$ in $50 \mu \mathrm{L}$ PBS) were injected directly into the tail of the pancreas under anesthesia. Dabigatran (BIBR1048, at a dose of $80 \mathrm{mg} / \mathrm{kg}$ in $0.5 \%$ Natrosol solution, as suggested by the manufacturer and shown to be well tolerated in mice [25-28]) or $0.5 \%$ Natrosol solution (placebo control) was administered by oral gavage twice daily starting from $7 \mathrm{~d}$ after tumor cell injection. If indicated, mice were treated with gemcitabine $(100 \mathrm{mg} / \mathrm{kg}$ in PBS, intraperitoneal) or PBS (control) starting from $7 \mathrm{~d}$ after tumor cell injection, and this treatment was repeated twice weekly. Mice were evaluated for changes in body weight and signs of discomfort or morbidity, and they were euthanized 3 or 5 (gemcitabine experiment) wks after tumor cell injection.

\section{(Immuno)histochemistry}

Histological examination was performed essentially as previously described $(29,30)$. Briefly, the excised tumor was fixed in formalin, embedded in paraffin and $4 \mu \mathrm{m}$ thick slides were subsequently deparaffinized, rehydrated and washed in deionized water. Slides were stained with hematoxylin and eosin (H\&E) according to routine procedures. For immunohistochemistry of Ki67, F4/80 (also known as EGF-like module-containing mucin-like hormone receptor-like 1), arginase 1 (Arg1), $\alpha$-smooth muscle actin ( $\alpha$-SMA) and cluster of differentiation 31 (CD31; also known as platelet endothelial cell adhesion molecule), endogenous peroxidase activity was quenched with $0.3 \%$ hydrogen peroxide in methanol for 15 or $20(\mathrm{~F} 4 / 80) \mathrm{min}$ at room temperature, and antigen retrieval was performed for $10 \mathrm{~min}$ (Ki67, $\alpha$-SMA and CD31) or $20 \mathrm{~min}$ (F4/80 and Arg1) at $96^{\circ} \mathrm{C}$ in $10 \mathrm{mM}$ sodium citrate buffer, $\mathrm{pH}$ 6.0. Next, slides were blocked for $15 \mathrm{~min}$ with Ultra V block (Thermo Scientific) or (for $\alpha$-SMA) 30 min with 5\% normal goat serum. Primary antibodies against Ki67 (1:500, clone Sp6; Neomarkers), Arg1 (1:2000, a gift from Prof W Lamers), F4/80 (1:400, clone:CI:A3-1; Serotec) or a-SMA (1:800, clone 1A4; Santa Cruz) were incubated overnight at $4^{\circ} \mathrm{C}$. For CD31, the primary antibody (1:1000; sc-1506-R; Santa Cruz Biotechnology) was incubated for $90 \mathrm{~min}$ at room temperature. Slides were subsequently incubated with appropriate horseradish peroxidaseconjugated secondary antibodies, and 3,3'-diaminobenzidine staining was used to visualize peroxidase activity. Slides were photographed with a microscope equipped with a digital camera (Leica CTR500, Leica Microsystems). CD31positive vessels were counted in 10 different fields per slide at $400 \times$ magnification. F4/80, Arg1, Ki67 and $\alpha$-SMA staining was analyzed with ImageJ and expressed as percentage of surface area. Depending on tumor size, an average of 10 to 30 pictures (random field) at $200 \times$ magnification per tumor were used for analysis. 


\section{Cell Viability and Proliferation}

Cell viability was measured using 3-(4,5-dimethylthiazol-2-yl)-2,5-diphenyltetrazolium bromide reduction assays as previously described (11). Cell proliferation was measured using a cell proliferation enzyme-linked immunosorbent assay 5-bromo-2'-deoxyuridine (Roche) as previously described (31).

\section{Statistical Analysis}

Results are presented as means \pm standard error of the mean. Differences between multiple groups were analyzed by two-way analysis of variance and/or Tukey $t$ test using GraphPad Prism with $P<.05$ considered significant $\left({ }^{*} P<.05 ; * * P<.001\right.$ and $* * * P<.0001)$.

All supplementary materials are available online at www.molmed.org.

\section{RESULTS}

\section{Influence of Dabigatran on Pancreatic Cancer Growth and Tumor Cell Dissemination}

To assess the importance of thrombin in pancreatic cancer, we employed dabigatran etexilate in a murine orthotopic pancreatic cancer model, in which Panc02 pancreatic cancer cells were injected into the pancreases of $\mathrm{C} 57 \mathrm{Bl} / 6$ mice. Three weeks after tumor cell inoculation, dabigatran-treated mice showed signs of bleeding and mice were consequently euthanized. As shown in Figure 1A, the peritoneal cavities of dabigatran-treated mice were indeed filled with blood, as opposed to the peritoneal cavities of placebo-treated controls. Subsequent analysis of tumor weight and volume showed that dabigatran treatment did not affect primary tumor growth (average weight and volume $0.74 \pm 0.27 \mathrm{~g}$ and $1.42 \pm 0.81 \mathrm{~cm}^{3}$ versus $0.83 \pm 0.26 \mathrm{~g}$ and $1.81 \pm 0.72 \mathrm{~cm}^{3}$ for placebo- and dabigatran-treated mice, respectively; Figures 1B, C). In contrast, dabigatran treatment significantly increased tumor dissemination and subsequent growth on distant organs as compared with control mice (Figure 1D).

Histologic examination of H\&E-stained pancreatic cancer sections showed clear signs of intratumoral bleeding (i.e., the omnipresence of extravascular red blood cells) in the dabigatran group as opposed to the control group (Figure 2A), likely explaining the increase in tumor dissemination throughout the peritoneal cavity. Interestingly, bleeding in dabigatran-treated mice was accompanied by increased numbers of CD31-positive blood vessels in the primary tumors. Indeed, the number of CD31-positive vessels in dabigatran-treated mice (17.27 \pm 2.76 ) was 1.4 -fold higher than that in control mice $(12.38 \pm 2.89)$ (Figure 2B).

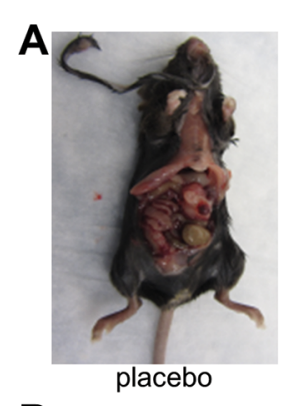

B

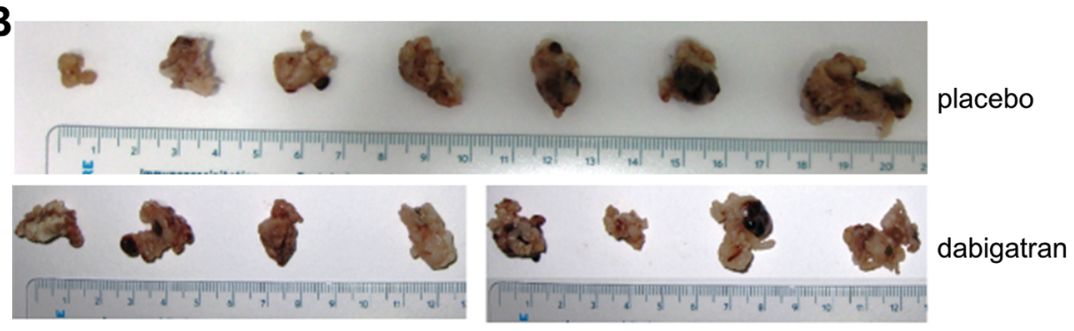

C
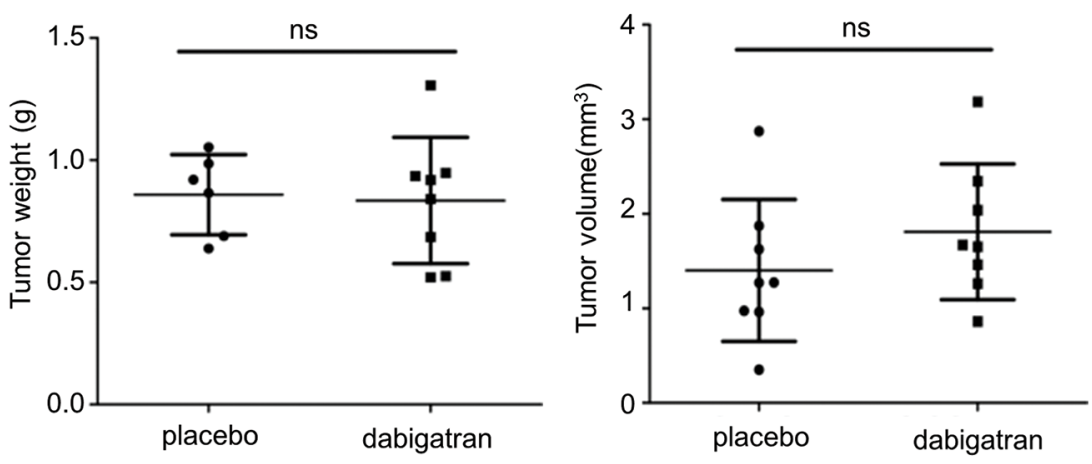

D

\begin{tabular}{c|c|c|c|c|c|c|} 
& spleen & liver & duodenum & colon/intestine & ovary & stomach \\
\hline placebo & $7 / 7$ & $1 / 7$ & $0 / 7$ & $1 / 7$ & $0 / 7$ & $0 / 7$ \\
\hline dabigatran & $8 / 8$ & $1 / 8$ & $3 / 8$ & $5 / 8$ & $6 / 8$ & $2 / 8$ \\
\hline
\end{tabular}

Figure 1. Influence of dabigatran monotherapy on pancreatic cancer growth and tumor cell dissemination. (A) Representative pictures of bleeding in the peritoneal cavity of dabigatran-treated mice at the moment of euthanization. (B) Pancreatic tumors derived 3 wks after tumor cell inoculation from mice after placebo or dabigatran treatment. (C) Weight and size of the tumors depicted in (A). Indicated is the mean \pm standard error of the mean (SEM) ( $n=7-8$ mice per group). NS $=$ not significant. (D) Number of mice with distant metastasis in the indicated organs at the time of euthanization (7-8 mice per group). 

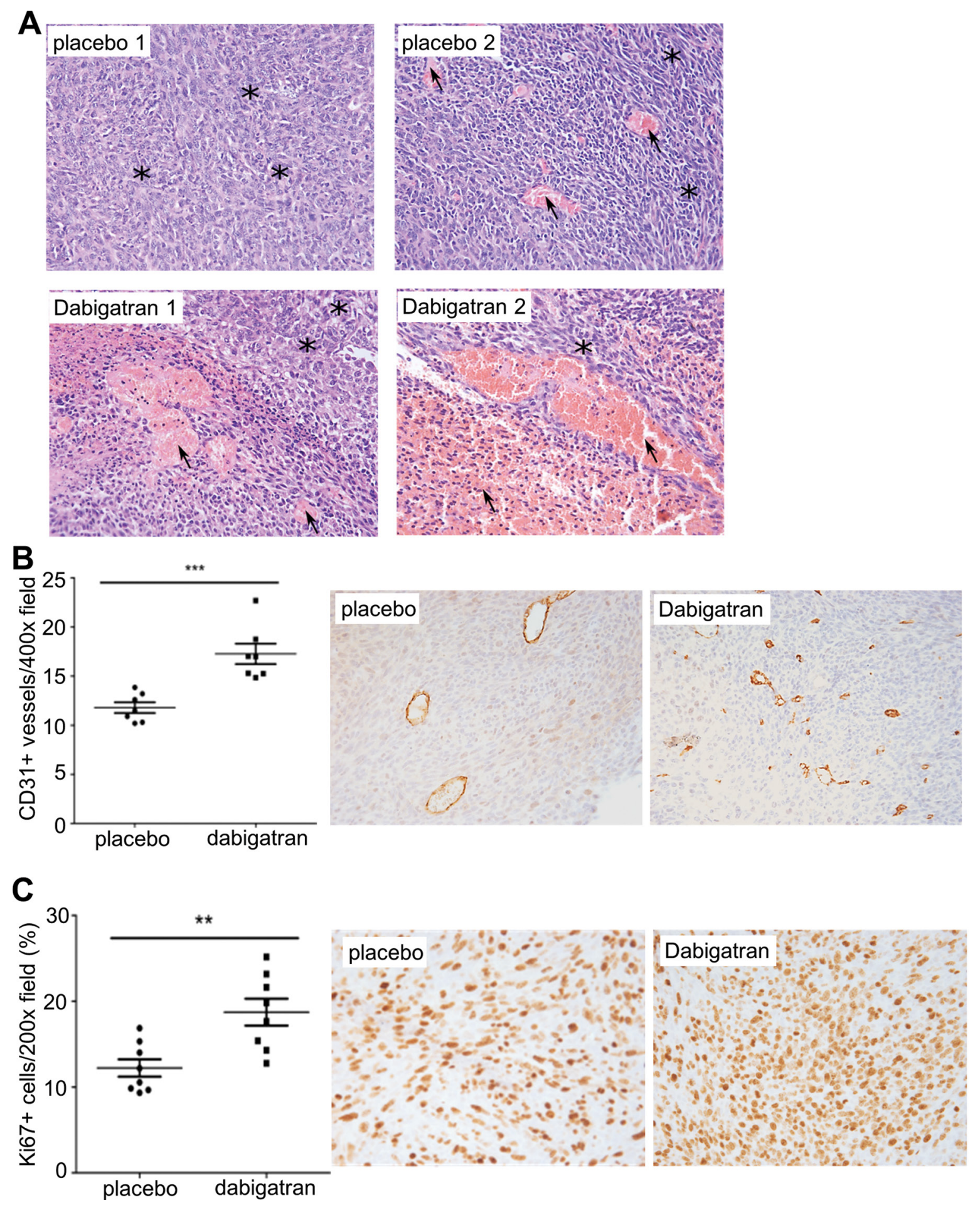

Figure 2. Dabigatran monotherapy is associated with angiogenesis and tumor cell proliferation. (A) H\&E-stained pancreatic cancer sections obtained from placebo or dabigatran-treated mice. Representative pictures were taken at magnification of $200 \times$, * indicates tumor tissue and arrow indicates red blood cells. (B and C) Paraffin sections obtained from placebo or dabigatran-treated mice stained for (B) CD-31 (blood vessel formation) and (C) Ki-67 (proliferation). Right panels show quantifications of the sections depicted in the left panels. Representative pictures were taken at $200 \times$ magnification. 
A

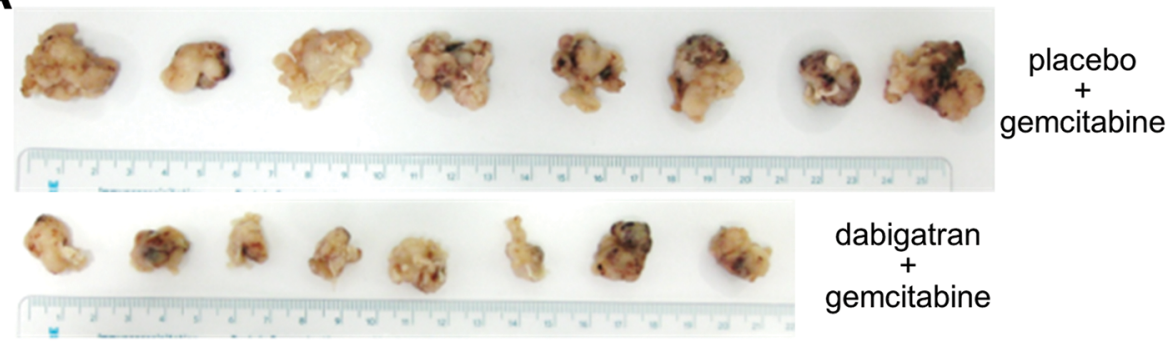

B
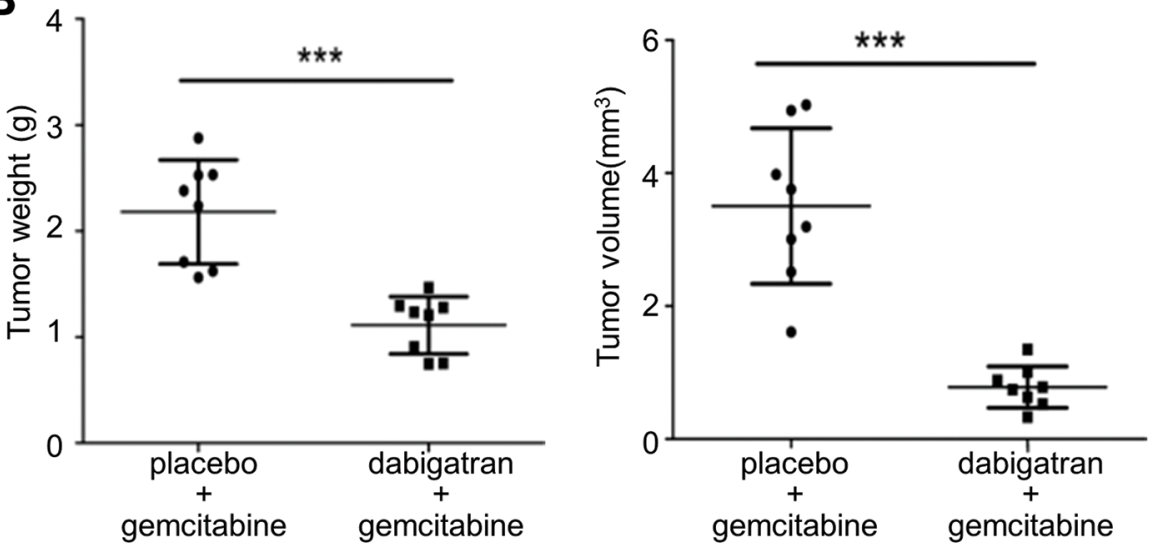

\begin{tabular}{l|c|c|c|c|c|c|} 
C & spleen & liver & colon/intestine & ovary & kidney & stomach \\
\hline $\begin{array}{l}\text { placebo + } \\
\text { gemcitabine }\end{array}$ & $8 / 8$ & $7 / 8$ & $1 / 8$ & $3 / 8$ & $5 / 8$ & $3 / 8$ \\
\hline $\begin{array}{l}\text { dabigatran + } \\
\text { gemcitabine }\end{array}$ & $5 / 8$ & $2 / 8$ & $0 / 8$ & $0 / 8$ & $1 / 8$ & $0 / 8$ \\
\hline
\end{tabular}

Figure 3. Effect of dabigatran in combination with gemcitabine on pancreatic cancer growth and tumor cell dissemination. (A) Pancreatic tumors derived 5 wks after tumor cell inoculation from gemcitabine- and dabigatran/gemcitabine-treated mice. (B) Weight and size of tumors depicted in (A). Indicated is the mean \pm SEM ( $n=8$ mice per group). ${ }^{* * *} P<.0001$. (C) Number of mice with distant metastasis in the indicated organs at the time of euthanization (8 mice per group).

The majority of vessels in the dabigatrantreated mice seemed aberrant and/or collapsed, however. As angiogenesis is pivotal for tumor cell proliferation, we consequently stained for the proliferation marker Ki67 and, as shown in Figure 2C, we indeed observed a significant increase in the number of Ki67-positive cells in dabigatrantreated mice as compared with control mice.

\section{Combination Therapy of Dabigatran and Gemcitabine in Pancreatic Cancer}

Gemcitabine has been used as a standard treatment in pancreatic cancer patients for many years (32). As shown in Supplementary Figure S1, gemcitabine reduces tumor weight and volume in our orthotopic model by around twoand three-fold, respectively. To assess whether thrombin modifies the intrinsic drug resistance of pancreatic cancers, mice with orthotopically injected pancreatic cancer cells were also treated with gemcitabine alone or with a combination of gemcitabine and dabigatran. As we did not observe any signs of bleeding in the gemcitabine/dabigatran group at the moment we had to euthanize the groups without gemcitabine treatment, we opted to euthanize the gemcitabine-treated mice at the predefined 5-wk time point based on pilot experiments showing only small tumors in gemcitabine-treated mice at the 3-wk time point. As shown in Figures 3A and $B$, mice treated with gemcitabine alone had primary tumors with an average weight of $2.18 \pm 0.49 \mathrm{~g}$ and a volume of $3.50 \pm 1.17 \mathrm{~cm}^{3}$. Interestingly, tumors in mice treated with both gemcitabine and dabigatran were significantly lighter $(1.11 \pm 0.27 \mathrm{~g})$ and smaller $\left(0.78 \pm 0.31 \mathrm{~cm}^{3}\right)$ than those in the gemcitabine group. In addition to reducing primary tumor growth, dabigatran co-treatment also reduces tumor cell spread throughout the peritoneal cavity. As shown in Figure 3C, the number of mice with tumor cells on distant organs was largely reduced in the gemcitabine/dabigatran group as compared with the gemcitabine group.

Dabigatran treatment does not lead to increased numbers of CD31-positive vessels in the primary tumors of mice treated with gemcitabine (Figure 4A). Moreover, the CD31 staining also showed less aberrant or collapsed vessels in the dabigatran/gemcitabine group than in the dabigatran group (Figure 2B). Moreover, the number of proliferating Ki67-positive cells was clearly reduced in mice treated with both dabigatran and gemcitabine as compared with mice treated with gemcitabine alone (Figure 4B).

\section{Effect of Dabigatran on Macrophage Recruitment and Fibroblast Activation in Pancreatic Cancers}

Our previous data suggest that PAR-1 drives gemcitabine resistance in pancreatic cancer by modulating macrophage 
A

\section{B}

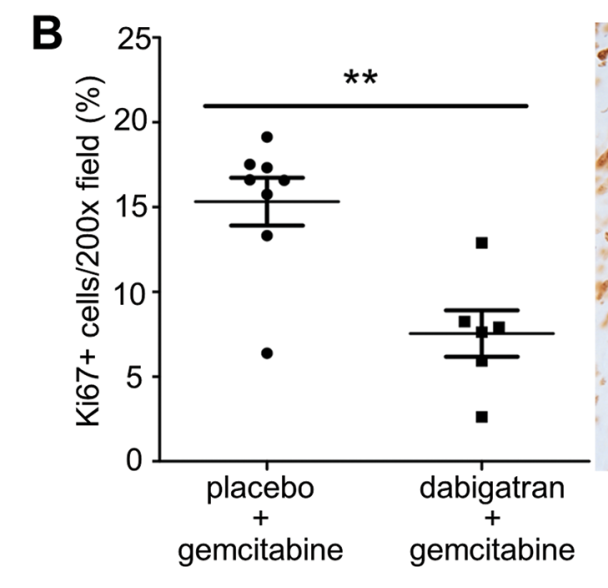

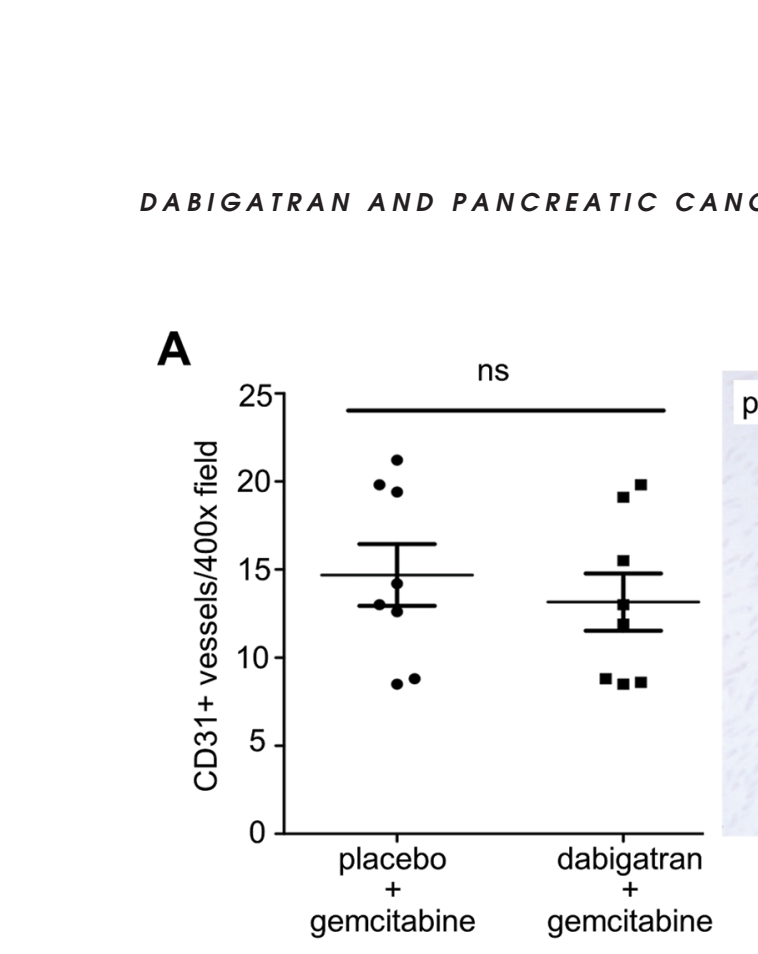
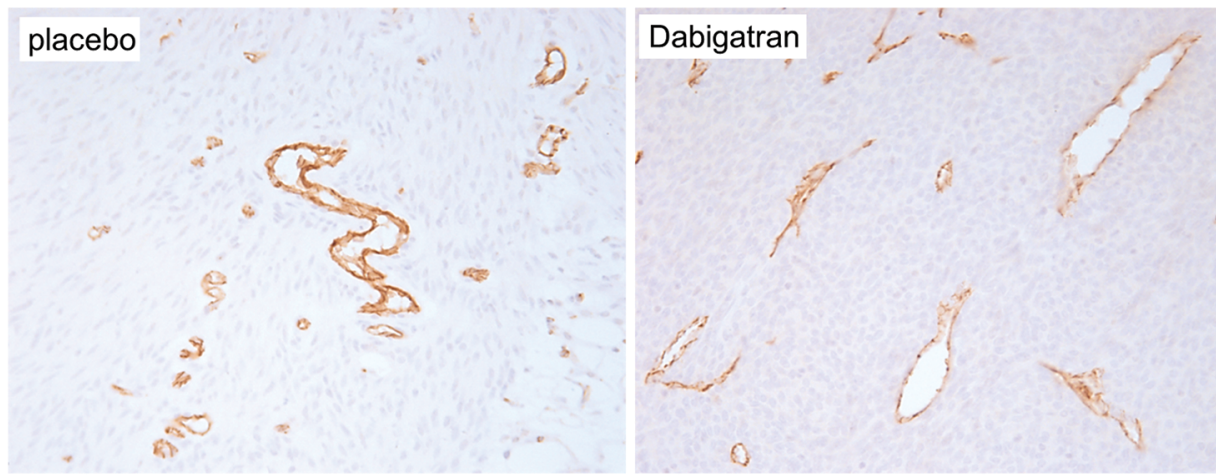

.

\footnotetext{
.
}

Figure 4. Dabigatran treatment limits angiogenesis and tumor cell proliferation in mice treated with gemcitabine. Paraffin sections obtained from tumors of gemcitabine- or dabigatran/gemcitabine-treated mice stained for (A) CD-31 and (B) Ki67. Right panels show quantifications of the sections depicted in the left panels. NS = not significant. ${ }^{* *} P<.001$. Representative pictures were taken at $200 \times$ magnification.

recruitment into the tumor (11). Consequently, we next analyzed the number of tumor-associated macrophages in dabigatran-treated and control mice to assess whether the increased gemcitabine sensitivity could relate to reduced macrophage recruitment. As shown in Figure 5A, however, the number of F4/80 positive cells was not influenced by dabigatran treatment. Moreover, dabigatran treatment also did not modify macrophage polarization, as evident from a similar percentage of Arg1 (M2 polarization marker) positive cells in dabigatran-treated and control mice (Figure 5B).

Drug resistance of pancreatic cancer also has been suggested to rely on the desmoplastic reaction $(22,33)$ and cancer-associated fibroblasts promote tumor progression (34). As thrombin is a key factor in fibroproliferative disease (35), thrombin-driven fibroblast activation may explain the differences seen in gemcitabine efficiency. As shown in Figure 5C, however, the number of matrix-producing $\alpha \mathrm{SMA}$-positive fibroblasts is not different in dabigatran-treated or control mice.

\section{Gemcitabine Sensitivity of Panc02 Cells In Vitro}

An alternative explanation for the observed increased gemcitabine sensitivity in dabigatran-treated mice could be that thrombin directly affects the cytotoxicity of gemcitabine. As a readout of drug efficiency, we used both 3-(4,5-dimethylthiazol-2-yl)-2, 5-diphenyltetrazolium bromide and 5-bromo-2'-deoxyuridine assays to illustrate cell viability and proliferation. As shown in Figure 6A, however, thrombin stimulation of Panc02 cells $(0.1-1 \mathrm{U} / \mathrm{mL})$ does not affect gemcitabine-induced cell death. Alternatively, thrombin could act upon tumor-associated fibroblasts, thereby inducing gemcitabine resistance of Panc02 cells. Medium transfer of primary tumor-associated fibroblasts stimulated with thrombin, however, did not modify gemcitabine-induced cytotoxicity (Figure 6B). Importantly, gemcitabine induces cytotoxicity by inhibiting DNA 
A
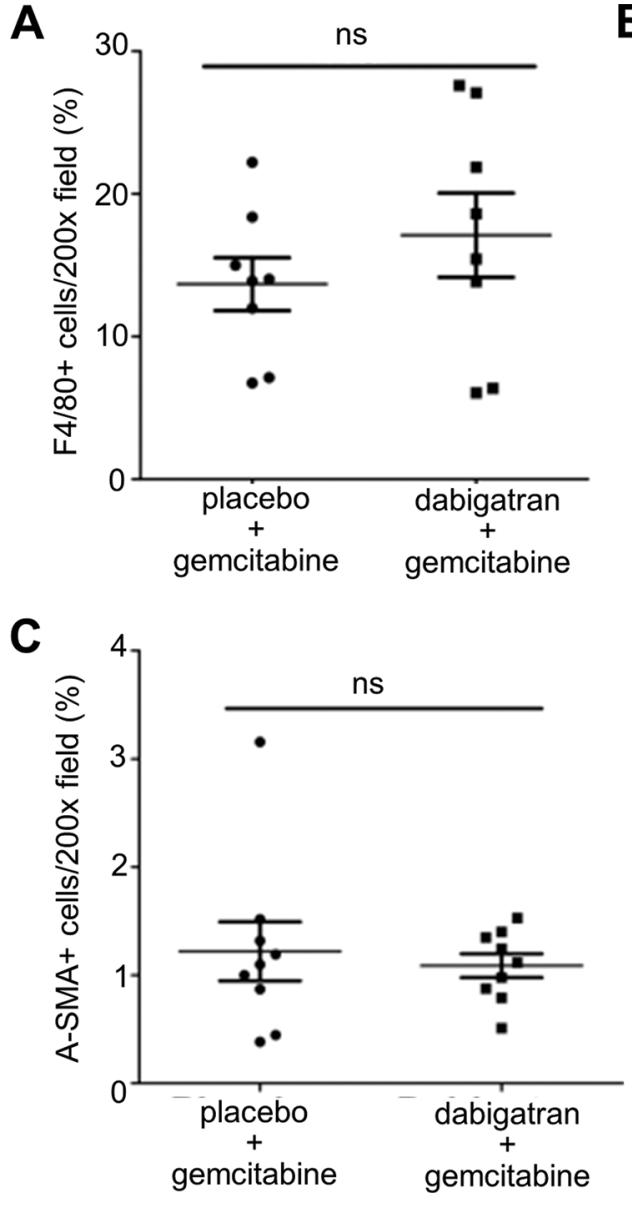

B

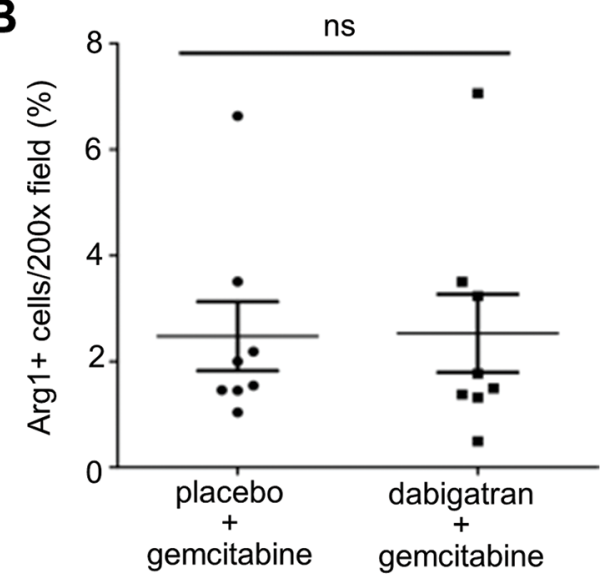

tumor growth in $75 \%$ of the mice (11), whereas all dabigatran-treated mice showed pancreatic tumors (Figure 1) already at 3 wks. The most likely explanation for the limited effect of dabigatran may be that thrombin is not the main PAR-1 agonist in the setting of pancreatic cancer. Next to thrombin, several PAR-1 agonists, such as (among others) matrix metalloproteinase-1 (also known as interstitial collagenase) (36), prohibitins (37), granzyme K (38), kallikrein-related peptidase-4 (39) and serine protease 3 (also known as trypsin 3) (40) were recently identified. Serine protease 3 could be especially relevant, as it seems to play an important role in the progression, metastasis and prognosis of human pancreatic cancer (41). Alternatively, thrombin inhibition might not be as effective as PAR-1 deficiency, as drug delivery is notoriously difficult in pancreatic cancer (42). Finally, one could argue that the observed difference between stromal PAR-1 deficiency and dabigatran treatment could be due to the fact that dabigatran also targets thrombin-induced effects on tumor cells. However, thrombin is mainly considered a driver of tumor cell proliferation (43) and, in the setting of pancreatic cancer, enhances the adhesion of pancreatic cancer cells to the extracellular matrix and/or endothelium (14).

Dabigatran treatment is associated with increased numbers of blood vessels in primary tumors (Figure 2). This is somewhat puzzling, as thrombin is known to enhance angiogenesis (44), and anticoagulants inhibit tumor cell-mediated angiogenesis (45). Interestingly, however, low thrombin levels (i.e., $0.1-0.3 \mathrm{U} /$ $\mathrm{mL}$ ) more potently induce angiogenesis as compared with high (i.e., $1 \mathrm{U} / \mathrm{mL}$ ) thrombin levels (46), suggesting that residual thrombin activity in dabigatran-treated mice might actually induce angiogenesis. In line, some studies do show that thrombin inhibits angiogenesis in vitro (47), whereas both FX and FXa possess anti-angiogenic properties in vitro in zebra fish intersegmental vasculature formation and in chick embryo chorioallantoic membrane assays (48). 

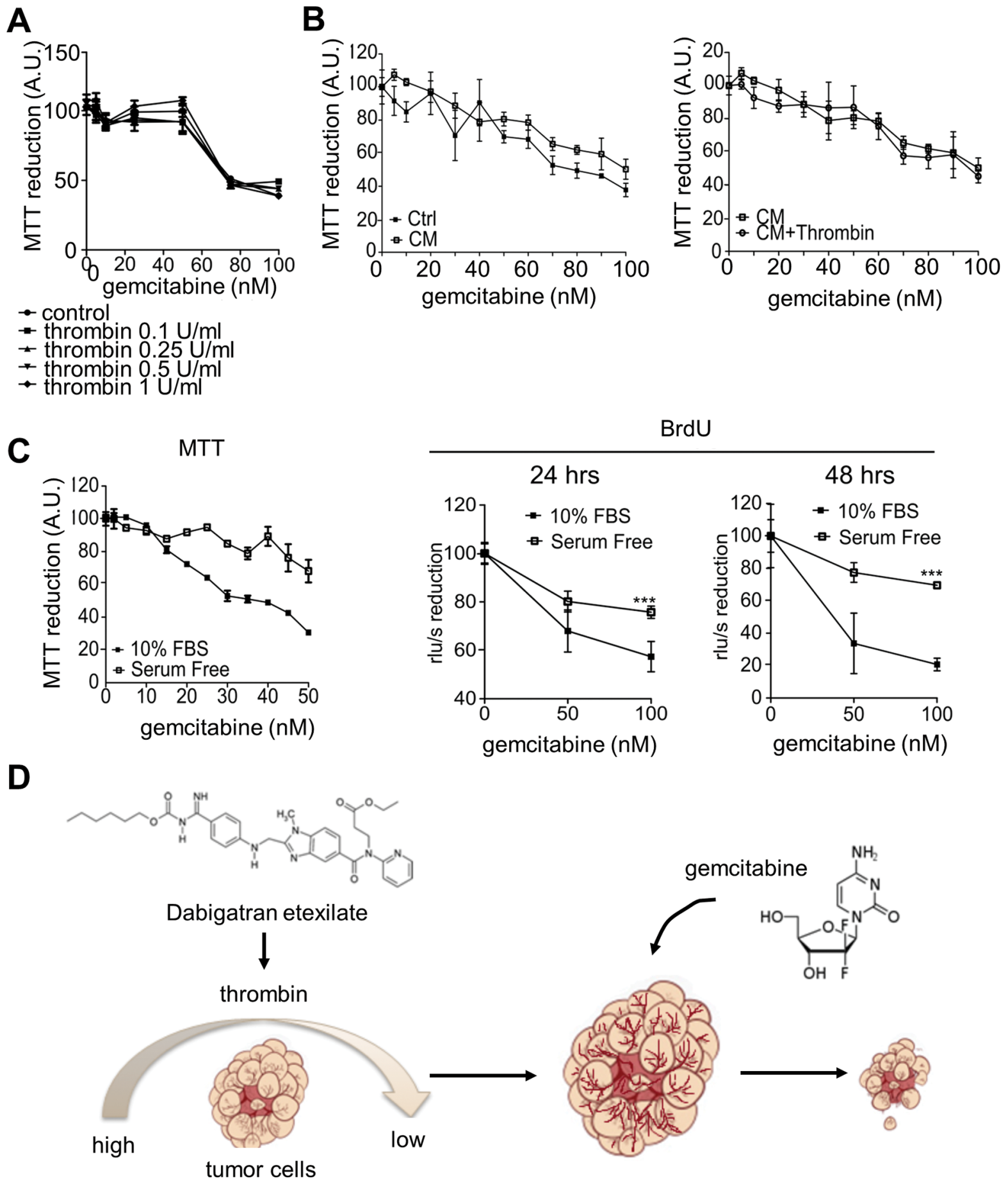

Figure 6. Gemcitabine sensitivity of Panc02 cells in vitro. (A) Effect of thrombin stimulation on gemcitabine sensitivity of Panc02 pancreatic cancer cells. (B) Conditioned medium (CM) collected from tumor-associated fibroblasts did not affect gemcitabine efficiency (left panel), and thrombin-stimulated tumor-associated fibroblasts did not enhance gemcitabine resistance (right panel). (C) Effect of gemcitabine on cell viability (left panel) and proliferation (right panel) of Panc02 cells in the presence or absence of growth factors. Shown are the means \pm SEM of three experiments performed in sixplo. (D) Proposed mechanism by which dabigatran potentiates gemcitabine-induced growth inhibition of pancreatic cancers. Dabigatran reduces thrombin production, which (1) subsequently leads to increased angiogenesis and (2) subsequently increases proliferation of tumor cells. Highly proliferative tumor cells are finally efficiently killed by gemcitabine (3). 
We previously showed that macrophages play a key role in PAR-1-dependent drug resistance of pancreatic cancer (11). Dabigatran treatment, however, does not affect macrophage recruitment (Figure 5A, B) and thrombin-dependent drug resistance thus seems macrophage independent. Alternatively, tumorassociated fibroblasts could modify drug resistance (49-51) in a thrombin-dependent manner, although PAR-1 deficiency did not affect fibroblast numbers or drug sensitivity (11). Dabigatran treatment, however, did not modify the number of SMA-positive fibroblasts within the tumor (Figure 5C), nor did thrombin limit tumor-associated fibroblast-induced resistance in vitro (Figures 6A, B). Consequently, we hypothesize that dabigatraninduced proliferation, as evidenced by increased numbers of Ki67-positive cells (Figure 2C), explains the increased gemcitabine sensitivity (Figure 6D). Indeed, gemcitabine blocks DNA synthesis and is more effective on rapidly proliferating cells (52). In line, gemcitabine cytotoxicity is increased in highly proliferative Panc02 as compared with growth factor-depleted low-proliferative Panc02 cells (Figure 6C).

Tumor cell dissemination throughout the peritoneal cavity is increased in dabigatran-treated mice as compared with control mice (Figure 1). The increase of aberrant or collapsed vessels in tumors of dabigatran-treated mice combined with bleeding in these mice likely explains the increased dissemination. Importantly, dabigatran-induced bleeding seems specific for pancreatic cancer, as the dabigatran dose used (i.e., $80 \mathrm{mg} / \mathrm{kg}$, twice daily) is well tolerated and did not induce bleeding in an ovarian cancer model (25), whereas longterm treatment with a dose of $140 \mathrm{mg} / \mathrm{kg}$ (administered via oral gavage) is safe and did not induce bleeding in models of allergic lung inflammation (26), highfat-diet-induced fatty liver disease (27) and glial activation (28). Dabigatraninduced bleeding in our orthotopic pancreatic cancer model is probably due to the fact that orthotopically grown pancreatic tumors are already prone to bleed at end-stage disease when primary tumors are large (personal observation). Notably, combined gemcitabine/dabigatran treatment does not induce bleeding complications and results in reduced tumor cell dissemination.

Several clinical studies have evaluated the potential clinical efficacy of anticoagulants in pancreatic cancer patients. In a nonrandomized study, the addition of low-molecular-weight heparin (LMWH) to standard gemcitabine/ cisplatinum chemotherapy significantly improved survival in patients with locally advanced or metastatic pancreatic carcinoma (53). The total response rate was almost $60 \%$ for patients treated with LMWH, compared with only $12 \%$ for patients treated with chemotherapy only. The response of pancreas cancer patients to LMWH treatment was confirmed in a retrospective analysis of patients who received chemotherapy for advanced pancreatic adenocarcinoma (54). The median survival of patients with metastatic disease increased from 3.8 to 6.6 months due to treatment. LMWH treatment had no significant effect on patients without metastatic disease (median survival of 10.3 and 8.3 months for LMWH-treated versus control patients). Contrary to this study suggesting that anticoagulants may increase the overall survival of pancreatic cancer patients, a recent randomized controlled clinical trial did not show any benefit of LMWH in pancreatic cancer patients (55). The clinical relevance of anticoagulants and the importance of thrombin in pancreatic cancer are therefore still under debate. The current study suggests that thrombin inhibition only limits pancreatic cancer progression in combination with gemcitabine, and it would be interesting to address whether such a relation also holds true in the randomized controlled clinical trial.

In the current manuscript, we employed an orthotopic pancreatic cancer model in which murine pancreatic cancer cells were grafted into the pancreases of immune-competent mice. This syngeneic orthotopic model offers tissue-specific pathology and is generally deemed more clinically relevant compared with xenograft models, in which human tumor cells are transplanted as xenografts into immunodeficient mice $(56,57)$. As elegantly indicated in a recent review (58), murine models using human cells have shortcomings, such as harboring impaired immune infiltrates (e.g., lymphocytes, natural killer cells). This is particularly important, as a complex relationship exists between the immune system and pancreatic cancer development, and these complex interactions have important implications for disease progression and control $(59,60)$. For example, CD8 + T cells and Th1-polarized CD4 + T cells mediate tumor protection in murine pancreatic cancer models and are associated with prolonged survival in humans $(61,62)$. Likewise, blocking cytotoxic CD8 + anti-tumor responses by myeloidderived suppressor cells significantly accelerates pancreatic cancer growth (63). In contrast, antigen-restricted Th2deviated CD4 + T cells strongly promote pancreatic cancer progression in mice (64). Accordingly, intratumoral CD4 + Th2 cell infiltrates correlate with reduced survival in human pancreatic cancer $(61,62)$. Next to a dysfunctional immune system, xenografts using human cells possess certain aberrant cross-species ligand-receptor interactions (e.g., hepatocyte growth factor-c-mesenchymalepithelial transition) (65). Importantly, human-mouse species differences have also been described for interactions of coagulation factors (66), which suggests that murine xenografts may be preferred over human xenografts when targeting the coagulation cascade.

\section{CONCLUSION}

Dabigatran-dependent thrombin inhibition potentiates gemcitabineinduced growth inhibition and tumor cell dissemination of pancreatic cancer. In the absence of gemcitabine, however, dabigatran treatment led to intratumoral bleeding and consequent increased tumor cell dissemination. 


\section{ACKNOWLEDGMENTS}

Dabigatran was kindly provided by Dr. Ashley Goss from the CardioMetabolic Disease Research department of Boehringer Ingelheim Pharmaceuticals. This study is supported by grants from the Dutch Cancer Foundation (2009-4324 and 2014-6782).

\section{DISCLOSURE}

The authors declare they have no competing interests as defined by Molecular Medicine or other interests that might be perceived to influence the results and discussion reported in this paper.

\section{REFERENCES}

1. Stathis A, Moore MJ. (2010) Advanced pancreatic carcinoma: current treatment and future challenges. Nat. Rev. Clin. Oncol. 7:163-72.

2. Gudjonsson B. (2002) Survival statistics gone awry: pancreatic cancer, a case in point. J. Clin. Gastroenterol. 35:180-84.

3. Ghaneh P, Costello E, Neoptolemos JP. (2007) Biology and management of pancreatic cancer. Gut. 56:1134-52.

4. Cardenes HR, Chiorean EG, Dewitt J, Schmidt M, Loehrer P. (2006) Locally advanced pancreatic cancer: current therapeutic approach. Oncologist. 11:612-23.

5. Vu TK, Hung DT, Wheaton VI, Coughlin SR. (1991) Molecular cloning of a functional thrombin receptor reveals a novel proteolytic mechanism of receptor activation. Cell. 164:1057-68.

6. Salah Z, et al. (2012) Regulation of human protease-activated receptor 1 (hPar1) gene expression in breast cancer by estrogen. FASEB J. 26:2031-42.

7. Nierodzik ML, Karpatkin S. (2006) Thrombin induces tumor growth, metastasis, and angiogenesis: Evidence for a thrombin-regulated dormant tumor phenotype. Cancer Cell. 10:355-62.

8. Cisowski J, et al. (2011) Targeting proteaseactivated receptor-1 with cell-penetrating pepducins in lung cancer. Am. J. Pathol. 179:513-23.

9. Diaz J, et al. (2012) Progesterone promotes focal adhesion formation and migration in breast cancer cells through induction of proteaseactivated receptor-1. J. Endocrinol. 214:165-75.

10. Zhu L, et al. (2012) Cooperation of proteaseactivated receptor 1 and integrin alphanubeta5 in thrombin-mediated lung cancer cell invasion. Oncol. Rep. 28:553-60.

11. Queiroz KC, et al. (2014) Protease-activated receptor-1 drives pancreatic cancer progression and chemoresistance. Int. J. Cancer. 135:2294-304.

12. Schmidlin F, Bunnett NW. (2001) Proteaseactivated receptors: how proteases signal to cells. Curr. Opin. Pharmacol. 1:575-82.
13. Macfarlane SR, Seatter MJ, Kanke T, Hunter GD, Plevin R. (2001) Proteinase-activated receptors. Pharmacol. Rev. 53:245-82.

14. Rudroff C, Striegler S, Schilli M, Scheele J. (2001) Thrombin enhances adhesion in pancreatic cancer in vitro through the activation of the thrombin receptor PAR 1. Eur. J. Surg. Oncol. 27:472-76.

15. Nutescu EA, Shapiro NL, Chevalier A. (2008) New anticoagulant agents: direct thrombin inhibitors. Cardiol. Clin. 26:169-87, v-vi.

16. Mungall D. (2002) BIBR-1048 Boehringer Ingelheim. Curr. Opin. Investig. Drugs. 3:905-07.

17. Gustafsson D. (2003) Oral direct thrombin inhibitors in clinical development. J. Int. Med. 254:322-34

18. Hankey GJ, Eikelboom JW. (2011) Dabigatran etexilate: a new oral thrombin inhibitor. Circulation. 123:1436-50.

19. Nagarakanti R, et al. (2011) Dabigatran versus warfarin in patients with atrial fibrillation: an analysis of patients undergoing cardioversion. Circulation. 123:131-36.

20. Eckman MH, Singer DE, Rosand J, Greenberg SM. (2011) Moving the tipping point: the decision to anticoagulate patients with atrial fibrillation. Circ. Cardiovasc. Qual. Outcomes. 4:14-21.

21. Ezekowitz MD, Aikens TH, Nagarakanti R, Shapiro T. (2011) Atrial fibrillation: outpatient presentation and management. Circulation. 124:95-99.

22. Scott KA, Amirehsani KA. (2015) Dabigatran etexilate: An alternative to warfarin for patients with nonvalvular atrial fibrillation. J. Am. Assoc. Nurse Pract. 27:190-96.

23. Ziske C, et al. (2008) Real-time high-resolution compound imaging allows percutaneous initiation and surveillance in an orthotopic murine pancreatic cancer model. Pancreas. 36:146-52.

24. Dineen SP, et al. (2010) Smac mimetic increases chemotherapy response and improves survival in mice with pancreatic cancer. Cancer Res. 70:2852-61.

25. Alexander ET, Minton AR, Peters MC, van Ryn J, Gilmour SK. (2016) Thrombin inhibition and cisplatin block tumor progression in ovarian cancer by alleviating the immunosuppressive microenvironment. Oncotarget. 7:85291-305.

26. de Boer JD, et al. (2015) Effect of the oral thrombin inhibitor dabigatran on allergic lung inflammation induced by repeated house dust mite administration in mice. Am. J. Physiol. Lung Cell Mol. Physiol. 309:L768-75.

27. Kopec AK, et al. (2014) Thrombin inhibition with dabigatran protects against high-fat-diet-induced fatty liver disease in mice. J. Pharmacol. Exp. Ther. 351:288-97.

28. Marangoni MN, et al. (2016) Differential effects on glial activation by a direct versus an indirect thrombin inhibitor. J. Neuroimmunol. 297:159-68.

29. Borensztajn K, et al. (2010) Protease-activated receptor-2 induces myofibroblast differentiation and tissue factor up-regulation during bleomycininduced lung injury: potential role in pulmonary fibrosis. Am. J. Pathol. 177:2753-64.
30. Duitman J, et al. (2012) CCAAT/enhancerbinding protein delta facilitates bacterial dissemination during pneumococcal pneumonia in a platelet-activating factor receptor-dependent manner. Proc. Natl. Acad. Sci. USA. 109:9113-18.

31. Shi K, Queiroz KC, Stap J, Richel DJ, Spek CA. (2013) Protease-activated receptor-2 induces migration of pancreatic cancer cells in an extracellular ATP-dependent manner. J. Thromb. Haemost. 11:1892-902.

32. Cunningham D, et al. (2009) Phase III randomized comparison of gemcitabine versus gemcitabine plus capecitabine in patients with advanced pancreatic cancer. J. Clin. Oncol. 27:5513-18.

33. Whatcott CJ, Posner RG, Von Hoff DD, Han H. (2012) Desmoplasia and chemoresistance in pancreatic cancer. In: Pancreatic Cancer and Tumor Microenvironment. Grippo PJ, Munshi HG (eds.). Transworld Research Network, Trivandrum, India. Chapter 8 (https:/ / www.ncbi.nlm.nih.gov/ pubmed/22876390).

34. Hwang RF, et al. (2008) Cancer-associated stromal fibroblasts promote pancreatic tumor progression. Cancer Res. 68:918-26.

35. Chambers RC, Leoni P, Blanc-Brude OP, Wembridge DE, Laurent GJ. (2000) Thrombin is a potent inducer of connective tissue growth factor production via proteolytic activation of protease-activated receptor-1. J. Biol. Chem. 275:35584-91.

36. Goerge T, et al. (2006) Tumor-derived matrix metalloproteinase- 1 targets endothelial proteinaseactivated receptor 1 promoting endothelial cell activation. Cancer Res. 66:7766-74.

37. Zhang Y, Wang Y, Xiang Y, Lee W. (2012) Prohibitins are involved in protease-activated receptor 1-mediated platelet aggregation. J. Thromb. Haemost. 10:411-18.

38. Cooper DM, Pechkovsky DV, Hackett TL, Knight DA, Granville DJ. (2011) Granzyme K activates proteaseactivated receptor-1. PLoS One. 6:e21484.

39. Ramsay AJ, et al. (2008) Kallikrein-related peptidase 4 (KLK4) initiates intracellular signaling via protease-activated receptors (PARs). KLK4 and PAR-2 are co-expressed during prostate cancer progression. J. Biol. Chem. 283:12293-304.

40. Knecht W, et al. (2007) Trypsin IV or mesotrypsin and p23 cleave protease-activated receptors 1 and 2 to induce inflammation and hyperalgesia. J. Biol. Chem. 282:26089-100.

41. Jiang G, et al. (2010) PRSS3 promotes tumour growth and metastasis of human pancreatic cancer. Gut. 59:1535-44.

42. Neesse A, et al. (2011) Stromal biology and therapy in pancreatic cancer. Gut. 60:861-68.

43. Borensztajn KS, Spek CA. (2008) Proteaseactivated receptors, apoptosis and tumor growth. Pathophysiol. Haemost. Thromb. 36:137-47.

44. Tsopanoglou NE, Maragoudakis ME. (2009) Thrombin's central role in angiogenesis and pathophysiological processes. Eur. Cytokine Netw. 20:171-79. 
45. Battinelli EM, et al. (2014) Anticoagulation inhibits tumor cell-mediated release of platelet angiogenic proteins and diminishes platelet angiogenic response. Blood. 123:101-02.

46. Haralabopoulos GC, Grant DS, Kleinman HK, Maragoudakis ME. (1997) Thrombin promotes endothelial cell alignment in Matrigel in vitro and angiogenesis in vivo. Am. J. Physiol. 273: C239-45.

47. Wang B, Pearson T, Manning G, Donnelly R. (2010) In vitro study of thrombin on tubule formation and regulators of angiogenesis. Clin. Appl. Thromb. Hemost. 16:674-78.

48. Lange $S$, et al. (2014) Independent anti-angiogenic capacities of coagulation factors X and Xa. J. Cell Physiol. 229:1673-80.

49. Ruffell B, Affara NI, Coussens LM. (2012) Differential macrophage programming in the tumor microenvironment. Trends Immunol. 33:119-26.

50. Fischer C, et al. (2007) Anti-PLGF inhibits growth of $\operatorname{VEGF(R)-inhibitor-resistant~tumors~without~}$ affecting healthy vessels. Cell. 131:463-75.

51. Zhang W, et al. (2010) Depletion of tumorassociated macrophages enhances the effect of sorafenib in metastatic liver cancer models by antimetastatic and antiangiogenic effects. Clin. Cancer Res. 16:3420-30.

52. Zeuner A, et al. (2014) Elimination of quiescent/ slow-proliferating cancer stem cells by Bcl-XL inhibition in non-small-cell lung cancer. Cell Death Differ. 21:1877-88.

53. Icli F, et al. (2007) Low molecular weight heparin (LMWH) increases the efficacy of cisplatinum plus gemcitabine combination in advanced pancreatic cancer. J. Surg. Oncol. 95:507-12.

54. von Delius S, et al. (2007) Effect of lowmolecular-weight heparin on survival in patients with advanced pancreatic adenocarcinoma. Thromb. Haemost. 98:434-39.

55. van Doormaal FF, et al. (2011) Randomized trial of the effect of the low molecular weight heparin nadroparin on survival in patients with cancer. J. Clin. Oncol. 29:2071-76.

56. Qiu W, Su GH. (2013) Development of orthotopic pancreatic tumor mouse models. Methods $\mathrm{Mol}$. Biol. 980:215-23.

57. Partecke LI, et al. (2011) A syngeneic orthotopic murine model of pancreatic adenocarcinoma in the C57/BL6 mouse using the Panc02 and 6606PDA cell lines. Eur. Surg. Res. 47:98-107.

58. Hwang CI, Boj SF, Clevers H, Tuveson DA. (2016) Preclinical models of pancreatic ductal adenocarcinoma. J. Pathol. 238:197-204.

59. Daley D, et al. (2016) $\gamma \delta$ T Cells Support Pancreatic Oncogenesis by Restraining $\alpha \beta$ T Cell Activation. . 166:1485-99, e15.

60. Inman KS, Francis AA, Murray NR. (2014) Complex role for the immune system in initiation and progression of pancreatic cancer. World J. Gastroenterol. 20:11160-81.

61. De Monte L, et al. (2011) Intratumor T helper type 2 cell infiltrate correlates with cancerassociated fibroblast thymic stromal lymphopoietin production and reduced survival in pancreatic cancer. J. Exp. Med. 208:469-78.

62. Fukunaga A, et al. (2004) CD8+ tumor-infiltrating lymphocytes together with CD4+ tumorinfiltrating lymphocytes and dendritic cells improve the prognosis of patients with pancreatic adenocarcinoma. Pancreas. 28:e26-e31.

63. Pylayeva-Gupta Y, Lee KE, Hajdu CH, Miller G, Bar-Sagi D. (2012) Oncogenic Kras-induced GM-CSF production promotes the development of pancreatic neoplasia. Cancer Cell. 21:836-47.

64. Ochi A, et al. (2012) MyD88 inhibition amplifies dendritic cell capacity to promote pancreatic carcinogenesis via Th2 cells. J. Exp. Med. 209:1671-87.

65. Frese KK, Tuveson DA. (2007) Maximizing mouse cancer models. Nat. Rev. Cancer 7:645-58.

66. Petersen LC, et al. (2005) Characterization of recombinant murine factor VIIa and recombinant murine tissue factor: a human-murine species compatibility study. Thromb. Res. 116:75-85.

Cite this article as: Shi K, et al. (2017) Dabigatran potentiates gemcitabine-induced growth inhibition of pancreatic cancer in mice. Mol. Med. 23:13-23. 